\title{
ARTICLE
}

\section{Eating disorders in children and adolescents}

\section{Dasha Nicholls \& Elizabeth Barrett}

Dasha Nicholls is a consultant child and adolescent psychiatrist at the Feeding and Eating Disorders Service, Great Ormond Street Hospital, London. Elizabeth Barrett is a consultant child and adolescent psychiatrist at South London and Maudsley NHS Foundation Trust. Correspondence Dr Dasha Nicholls, Consultant Child and Adolescent Psychiatrist \& Honorary Senior Lecturer, Feeding and Eating Disorders Service, Department of Child and Adolescent Mental Health, Great Ormond Street Hospital, London WC1N 3JH, UK. Email: d.nicholls@ucl.ac.uk

${ }^{\dagger}$ This is an updated version of a chapter published in Huline-Dickens S (ed) (2014) Clinical Topics in Child and Adolescent Psychiatry. RCPsych Publications.

\section{SUMMARY}

This article provides an overview of classification and outcome of eating disorders, before focusing on current evidence-based treatment for the two main disorders of anorexia nervosa and bulimia nervosa.

\section{LEARNING OBJECTIVES}

- Recognise the changing view of eating disorders with respect to DSM-5/ICD-11 classifications

- Recognise the differences between child and adolescent eating disorders and those of later onset

- Understand the comprehensive approach to the management and treatment of eating disorders in young people

\section{DECLARATION OF INTEREST}

None

Eating disorders in childhood and adolescence present a serious threat to health and wellbeing, including medical consequences ranging from growth delay to life-threatening effects of starvation and refeeding (Nicholls 2011a). Anorexia nervosa is frequently cited as the third most common chronic illness of adolescence (Lucas 1991).

\section{Setting the context: classification and epidemiology}

Historically, the eating disorders (for which the majority of literature uses DSM terminology) comprised anorexia nervosa, bulimia nervosa and eating disorders not otherwise specified (EDNOS), with separate classification of feeding disorders with onset before age 6 . The point at which the responsibility for food intake changes from parent to child is complex, and includes factors such as the accurate recognition of hunger and satiety, knowledge of food hygiene and handling, sensory integration of texture and smell, as well as an understanding of nutritional needs. Eating disturbance is a common manifestation of emotional issues (anxiety, mood) and a medium through which autonomy and control are negotiated. There are therefore many potential mechanisms through which the feeding to eating transition can be deviated or delayed.

The revised classification of feeding and eating disorders in DSM-5 (American Psychiatric Association 2013) and that proposed for ICD-11 recognises this continuous process. This is an important conceptual shift. Key changes for the classification of eating disorders (Uher 2012) can be summarised as follows.

- The merging of feeding and eating disorders into a single grouping with categories applicable across age groups.

- Diagnosis can be made on the basis of behaviours (e.g. parental report of excessive exercise) that indicate fear of weight gain or other underlying fears or beliefs.

- Broadening of the criteria for the diagnosis of anorexia nervosa and removing the requirement for amenorrhoea; extending the weight criterion to any significant underweight; and extending the cognitive criterion to include developmentally and culturally relevant presentations.

- Reduction of the frequency requirement to meet diagnostic thresholds for binge eating and vomiting.

- Inclusion of binge eating disorder as a specific category defined by subjective or objective binge eating in the absence of regular compensatory behaviour.

- Introduction of a new term, 'avoidant/restrictive food intake disorder' (ARFID), to classify restricted food intake in children or adults that is not accompanied by psychopathology related to body weight and shape (Bryant-Waugh 2010). This category replaces the current diagnosis of 'feeding disorder of infancy and early childhood'. No age criterion is included for ARFID, which, to meet the clinical threshold, requires nutritional impairment or social or psychological impact of a highly restricted diet on development and/or on family function. Individuals with this disorder are more likely to have other medically unexplained symptoms (MUS), or comorbid medical or neurodevelopmental disorders.

- 'Other specified feeding and eating disorder' (OSFED) replaces EDNOS for cases of atypical anorexia nervosa, atypical bulimia nervosa, 
atypical binge eating disorder, purging disorder and night eating syndrome.

An overall aim is to minimise use of the catchall diagnosis of 'unspecified feeding and eating disorder'.

Research using the new criteria is as yet sparse, but in a recent US study utilising the DSM-5 classification system, Stice et al (2013) followed up adolescent girls and reported that lifetime prevalence by age 20 was $0.8 \%$ for anorexia nervosa, $2.6 \%$ for bulimia nervosa, $3.0 \%$ for binge eating disorder, $2.8 \%$ for atypical anorexia nervosa, $4.4 \%$ for subthreshold bulimia nervosa, $3.6 \%$ for subthreshold binge eating disorder and $3.4 \%$ for purging disorder. This gives a combined prevalence for any eating disorder of $13.1 \%$ (5.2\% had anorexia nervosa, bulimia nervosa or binge eating disorder; $11.5 \%$ had feeding and eating disorders not elsewhere classified (FED-NEC)). Early-onset eating disorders therefore include anorexia nervosa, bulimia nervosa, binge eating disorder and ARFID, as well as a range of eating disorder-like presentations, currently termed atypical or unclassifiable. The DSM-5 work group decided, having reviewed the evidence, that obesity did not merit classification as an eating disorder (Marcus 2009).

\section{Outcomes and continuity into adult life}

Around 50\% of adolescents with anorexia nervosa respond to treatment within 12 months, and $75-80 \%$ achieve full or partial remission, regardless of treatment type (Lock 2010). This means, however, that $20-25 \%$ will still meet diagnostic criteria for anorexia nervosa after 2 years of treatment (Gowers 2007). Factors that have been associated with poorer outcome include admission to psychiatric hospital, eatingrelated obsessionality, eating disorder-specific psychopathology, binge eating and purging, older age and longer duration of illness (Gowers 2007; Le Grange 2012). For bulimia nervosa, around 40\% abstain from bingeing/purging after treatment, but relapse is common even by 6 months. Those with less severe eating concerns at baseline, lower baseline depression, fewer binge/purge episodes at presentation, and receiving family-based rather than individual treatment are more likely to be in remission at follow-up (Le Grange 2008).

Previous studies had suggested that outcome for childhood-onset eating disorders might be worse than for those with onset in adolescence. Data from a national sample of patients under 13 with eating disorders found that most were still in treatment at 1 year, and seven had been hospital in-patients for most of the year (Nicholls 2011b).
Keel \& Brown (2010) reviewed long-term outcome data from 26 studies. They found that most patients with anorexia nervosa achieved remission by 5 -year follow-up, those receiving inpatient treatment having a poorer prognosis. In a 10-year outcome study, most (69\%) participants met criteria for full recovery, but $51 \%$ had developed another axis I disorder (Herpertz-Dahlmann 2001). There was a significant association between psychiatric comorbidity and the outcome of the eating disorder. Recovered patients did not differ significantly from normal controls with regard to psychiatric morbidity and psychosocial functioning. Approximately 30\% of patients with bulimia nervosa and atypical bulimia nervosa remain ill 10-20 years after initial presentation, although symptom remission for binge and purge behaviour is high. Early adolescent bulimia nervosa carries a ninefold increase in risk for late adolescent bulimia nervosa and a twentyfold increase in risk for adult bulimia nervosa (Kotler 2001). Field et al (2012) report that girls with binge eating disorder were almost twice as likely as their unaffected peers to become overweight or obese or to develop depressive symptoms.

\section{Therapeutic models}

Although some advocate a medical illness model for eating disorders, most adopt a psychological formulation and treatment approach that considers developmental and systemic factors but still acknowledges individual genetic and neurobiological risk. Assumptions about the role of the family in aetiology have undergone enormous cultural shift away from blaming the parents to recognition of the importance of involving them in treatment and decision-making (Treasure 2007; Le Grange 2010). Controlled treatment trials in anorexia nervosa for patients under the age of 18 with an illness of less than 3 years' duration showed that family work was more effective at 5-year follow-up than individual therapy alone (Eisler 1997). The 'Maudsley model' of family-based treatment (Lock 2000) has since gained a considerable evidence base and is the best empirically supported treatment available to young people with anorexia nervosa (Lock 2015). It is essentially a structural model, with parents clearly in charge, and with externalisation of the anorexia as a key strategy. This involves conceptualising the illness (often given a name) as an entity provoking the child and family. The child, family and professionals are united in their struggle to disempower and banish the illness. For some patients, particularly those with obsessional features, systemic family therapy may be more 
effective (Agras 2014). A similar model for familybased treatment of bulimia nervosa has been described (Le Grange 2007a). No specific family therapy techniques have been described for the treatment of the 'atypical' eating disorders.

If a family-based approach is not possible or the young person does not respond to this approach, combination therapies or individual therapy models may be used. Individual therapy for young people would typically occur in conjunction with parental or family work, as the burden of change should not rest solely on the child. It can take the form of various models of psychotherapy, including adolescent-focused psychotherapy (Fitzpatrick 2010), interpersonal psychotherapy and psychodynamic psychotherapy. Parents need to support and respect the confidentiality of the sessions, and the therapist is accountable to the parents for the work being done, usually through periodic reviews or feedback. Behavioural techniques have a role in changing concrete, measurable aspects of behaviour, but have little impact on thoughts, beliefs and feelings. They are not much use in isolation, and at worst can be punitive. Cognitive-behavioural techniques, however, are a mainstay of treatment for bulimia nervosa, and can be adapted for use in anorexia nervosa and ARFID (Gowers 2009). For some types of ARFID, specific focus on systematic desensitisation using graded hierarchies and techniques addressing sensory sensitivities may also be necessary.

The 'therapeutic tool box' therefore needs to be varied and flexible, depending on the age, developmental stage and degree of cooperation of the child. For example, it is not helpful to sit in prolonged silence with a child who is unwilling or unable to engage in any communication. For a more detailed account of some of the therapeutic techniques of use in young people with eating disorders, see Watkins (2013) and Troupp (2013).

\section{Comprehensive approach}

A multidisciplinary approach is essential. Debate sometimes arises over which to tackle first: the eating behaviour or the emotional symptoms. Notably, growth failure, pubertal arrest and failure of bone accretion can have significant impact in as little as 6 months and so the multidisciplinary team needs not only to be aware of and identify all these competing problems, but also to find constructive ways to resolve them. Although motivation is an important factor to consider, therapeutic alliance does not predict outcome for anorexia nervosa (Pereira 2006), and young people recognise that some things do need to be done or decided against their will. It does matter how they are done, however, and respect and trust are important qualities in healthcare professionals managing young people with eating disorders (Tan 2003).

\section{Healthcare setting}

As a 2009 Cochrane review outlines, current policy in the UK and elsewhere places emphasis on the provision of mental health services 'in the least restrictive setting, whilst also recognising that some children will require inpatient care' (Shepperd 2009). A number of studies have emerged looking at health service context rather than the treatment itself. Of these, the most influential has been the Treatment Outcome for Child and Adolescent Anorexia Nervosa (TOuCAN) trial (Gowers 2007). This evaluated the clinical and cost-effectiveness of in-patient treatment compared with two variants of outpatient treatment: general (routine) treatment in child and adolescent mental health services (CAMHS) and a specialist adolescent eating disorders service for young people with anorexia nervosa. TOuCAN was a population-based, pragmatic randomised controlled trial (RCT) in England involving 215 young people (aged 12 to 18 , mean age 14 years 11 months) and 35 CAMHS teams. On follow-up at 1,2 and 5 years, using both standardised and self-report measures, there was significant improvement in all groups at each time point. The number achieving a good outcome was $19 \%$ at 1 year, $33 \%$ at 2 years and $64 \%$ (of those followed up) at 5 years. Analysis demonstrated no difference in efficacy of in-patient compared with out-patient treatment, or specialist compared with generalist treatment at any time point, when baseline characteristics were taken into account. The specialist out-patient programme was the dominant treatment in terms of incremental costeffectiveness. Of note, the specialist out-patient service used a largely cognitive-behavioural rather than family-based treatment model. Out-patient treatment had a higher probability of being more cost-effective than in-patient care (Byford 2007).

Overall, the TOuCAN study provided little support for lengthy in-patient psychiatric treatment on clinical or health economic grounds, and broadly supports existing guidelines on the treatment of anorexia nervosa, such as those published by the National Institute for Health and Care Excellence (NICE). These suggest that out-patient treatment should be offered to the majority, with in-patient treatment offered in rare cases (National Collaborating Centre for Mental Health 2004). Out-patient care, supported by brief (medical) in-patient management for correction of acute complications, may be a preferable 
approach, and this has since been corroborated by findings from two RCTs, one comparing in-patient treatment with day care (Herpertz-Dahlmann 2014) and the other exploring the optimal length of in-patient stay (Madden 2014). The health economic analysis and patients' and carers' views from the TOuCAN study suggested that anorexia nervosa should be managed in specialist services that have experience and expertise in its treatment.

House et al (2012) have taken these issues further, exploring the effect of direct access to specialist services on referral rates, admissions for in-patient treatment and continuity of care. In areas where specialist out-patient services were available, 2-3 times more cases were identified than in areas without such services, and there were significantly lower rates of admission for in-patient treatment and considerably higher consistency of care.

Together these studies support the development of specialist eating disorder services able to provide continuity of care where possible. To our knowledge, there is no research about feeding disorders services.

The most common reasons for hospital admission are listed in Box 1. Admission to a paediatric ward for primarily medical reasons tends to work successfully only in the context of continuity of paediatric care and where skilled psychiatric care is also available. Staff need to receive adequate training and health service managers should address barriers to joint working. Admission to psychiatric units can be for one of two reasons: first, acute admission for management of risks that cannot be addressed in the paediatric setting, for example suicidality, some extreme eating behaviours or need for restraint; and second, because out-patient therapy does not provide sufficiently intensive support for change. However, in the latter situation, the benefits of intensity of treatment need to be weighed up against the risks of removing young people from their family and school environment.

\section{Help for parents}

Mistrust of professionals and self-blame are common for parents. They may have been told explicitly that they are to blame, or have developed a sense of failure while attempting to overcome their child's difficulties. Clear information, both in the form of general literature and specifically about their own child's difficulties, can help in establishing trust. Additionally, eating disorders occur more often in some families. For example, there is a specific association between some ARFID presentations and maternal eating disorder, particularly food avoidance emotional disorder (FAED) (Watkins 2012). It is evident that parenting a child with a feeding or eating disorder is stressful, and many carers report mental health problems. Sometimes, parents develop a rejecting stance to their child or see the eating behaviour as a personal attack (indeed, their child may see it in that way too). Except where there are clear safeguarding concerns, it is unhelpful to the child or parents to conceptualise eating disorders in this way. Helping parents to bear the illness and the rejection that goes with it without rejecting their child is essential.

\section{Medication}

The evidence for use of medication in the treatment of childhood-onset eating disorders is limited, and the evidence for effectiveness is weak across the age range. Clinical guidelines (e.g. National Collaborating Centre for Mental Health 2004) regarding medications are therefore mostly based on consensus rather than strong research. What evidence there is mainly comes from studies in adults. Medications under consideration for anorexia nervosa are usually atypical antipsychotics, selective serotonin reuptake inhibitors (SSRIs) or micronutrient supplementation. For bulimia nervosa, studies have used SSRIs, other antidepressants and mood stabilisers, with reasonable effects on binge/purge frequency in adults. For binge eating disorder, medications that have been studied include SSRIs, serotonin-noradrenaline reuptake inhibitors (SNRIs), mood stabilisers and anti-obesity drugs. Pharmacotherapy for bulimia nervosa and binge eating disorder shows moderate effect sizes in

\section{B0X 1 Reasons for admission to hospital}

There are various reasons for admission other than being seriously medically unwell. It is important to clarify and agree the necessity for and the purpose of paediatric admission with the young person, family and team members.

Reasons for admission include:

- need for intravenous fluids to correct electrolyte abnormality

- refeeding for severe malnutrition

- management of physical complications of severe malnutrition and/or associated behaviours, such as electrolyte disturbance secondary to purging

- management of an acute medical illness unrelated to the eating disorder

- respite for parents

- assessment

- assessment and management of self-harm

- initiation or trial of medication

- management of uncontrolled compensatory behaviours (e.g. excessive exercise, vomiting)

- moving the young person towards taking responsibility for their own eating disorder when a family-based approach is not appropriate or effective and out-patient treatment would not be safe. 
adults, but generally low recovery rates. Anorexia nervosa is generally more treatment resistant.

Three RCTs have been conducted to date involving adolescents with anorexia nervosa:

- olanzapine $v$. placebo $(n=20)$ : preliminary findings did not support adjunctive olanzapine for underweight adolescent girls with anorexia nervosa (Kafantaris 2011)

- risperidone $v$. placebo $(n=40)$ : there was no benefit for the addition of risperidone during weight-restoration in adolescents with anorexia nervosa (Hagman 2011)

- quetiapine $v$. treatment as usual ( $n=33)$ : low-dose quetiapine in this open-label RCT resulted in both psychological and physical improvements, with minimal side-effects (Court 2010).

Antidepressants should be considered for patients who are clearly suffering from depression or obsessive-compulsive disorder, once the effects of malnutrition have abated. Given concerns about the safety of SSRIs in adolescents and children, caution is advised in using this class of medication for anorexia nervosa in this age group (Lock 2005a). The use of SSRIs is of proven efficacy in adults with bulimia nervosa and should be considered in adolescents (Flament 2012). Medication can also be considered as an adjunct to other therapies, particularly when it could enhance the capacity of the child to make use of other therapy. Anxiolytic medication may be a useful adjunct in the treatment of functional dysphagia. Medication should never be used in the absence of other treatments.

\section{Management and treatment: specific disorders}

\section{Anorexia nervosa}

\section{Responsibility}

Anorexia nervosa (Box 2) remains the psychiatric disorder with the highest mortality, and it can create enormous anxiety and conflict in personal and professional relationships. Issues of control and responsibility are central to the treatment of anorexia nervosa. With young people, these are further complicated by the need to determine to what extent they can be considered both to be competent and to have capacity to make decisions about treatment. Responsibility for recovery can be seen to lie with the child, the parents or the professionals. When the professionals take charge, this allows the parents and child to be relieved of the anxiety, but it can also reinforce the parents' sense of failure. Occasionally, it causes the parents to withdraw the young person from treatment if they are not in agreement with the professionals.
B0X 2 Features of anorexia nervosa

- Weight loss or maintenance of a significantly low expected weight for height and age, or failure to make weight gain during a growth period

- Fear of gaining weight or becoming fat, even though underweight

- Weight loss achieved by restriction of food intake and specific avoidance of 'fattening foods' and one or more of the following: self-induced vomiting, selfinduced purging, excessive exercise, use of appetite suppressants and/or diuretics

- Disturbance in the way the person experiences their body weight and shape (body image distortion), undue influence of body weight or shape on self-evaluation, morbid preoccupation with weight and shape

(Adapted from: World Health Organization 1992; American Psychiatric Association 2013

For these reasons, parents should be helped to take responsibility for managing the eating disorder, unless the age of the young person, urgency or other imperatives suggest otherwise. This means establishing parental control of food and fluid intake. The young person is encouraged to negotiate the 'how' of food intake, but not the 'whether'. For example, children may like their mother to sit with them during meals and hold their hand. Alternatively, they may prefer for food not to be discussed at meal times, and any extra calories to be made up in drink form at the end of the day. Whatever approach is taken, parents should apply it consistently, even if the parental couple are no longer living together. Once control of eating is established, other areas where the child can develop or regain control are negotiated. These form the first two stages of the family-based treatment model for anorexia nervosa.

In older adolescents moving towards independence, or when the illness is long-standing, it may be appropriate for more responsibility to lie with the young person, with parents moving to a supportive rather than responsible role. Techniques such as motivational interviewing or workbooks such as Hunger for Understanding (Nesbitt 2005) can engage the young person in the idea of change and recovery.

\section{Physical aspects}

Doctors have a central role in both the recognition and management of medically unstable children. Physical aspects of care can be both acute (e.g. medical compromise) and chronic (e.g. impaired growth and development) in nature. Differences in size and body composition put children at greater risk than adults for certain aspects of acute and 
chronic starvation. Prepubertal children become emaciated more quickly than adults, because of the relative deficiency of body fat, and they also dehydrate more quickly. Hudson et al (2012) looked in detail at children under 13 with eating disorders in the context of the British surveillance study. Over a third had medical instability at presentation (60\% had bradycardia, 54\% hypotension, 34\% dehydration, $26 \%$ hypothermia) and over $50 \%$ required admission shortly after diagnosis (73\% to a paediatric ward). Of those with medical instability, 41\% were not underweight. Peebles et al (2010), in a case-note review of 1310 females aged 8-19 years treated for anorexia nervosa, bulimia nervosa or EDNOS, found that the medical severity of those with EDNOS was intermediate on all parameters to that of those with anorexia nervosa or bulimia nervosa, but that those with partial bulimia nervosa or bulimia nervosa had longer QTc intervals and higher rates of additional medical complications at presentation than the other groups. Both studies provide evidence that anthropological indices alone are poor markers for medical instability, and clinical assessment is essential. The Junior MARSIPAN report (Royal College of Psychiatrists, 2012) outlines parameters for risk assessment in young people and gives guidance on laboratory tests and indicators of concern.

In terms of chronic sequelae of eating disorders, the most important are the impact on growth, pubertal development and bone health. Distinction should be made between children who are appropriately prepubertal and those in whom the onset of puberty has been delayed. Significant delay is usually defined as more than 2 standard deviations (s.d.) from the mean. Menses are deemed to be delayed if there is failure of onset within 4.5 years of the start of puberty, or by the time the girl has reached the chronological or bone age of 14 years. Onset of anorexia nervosa during puberty will result in pubertal arrest. Tanner staging, pelvic ultrasound appearances and discrepancy between bone age and chronological age can help in evaluating the degree of pubertal delay. Chronic physical illness or genetic factors resulting in pubertal delay need to be taken into account.

Height and weight should be plotted on standard growth charts for comparison with population norms and parental heights. Previous information about the child's growth will give a more complete picture. The 'growth spurt' in girls occurs at age 12 years (s.d. $=1.8$ years); thereafter, growth slows down and stature is only likely to be significantly affected if onset is post-menarcheal. Body mass index (BMI) is not a linear constant in childhood, so BMI needs to be adjusted for the young person's age and gender by use of a BMI chart or by calculating how far the BMI is from the median for age and gender. This can be expressed as a standard deviation score (SDS), or as a percentage of the median BMI:

\section{(BMI/median BMI for age and gender) $\times 100$}

(this is one way of calculating weight for height). Clinically, however, rate of weight loss may be more important than percentage BMI alone.

Osteoporosis is an established risk in adults with anorexia nervosa. In younger patients, the problem of bone loss is compounded by failure of bone accretion, especially important as adolescence is a critical time for bone accrual. Other contributing factors include hypogonadism and low oestradiol levels seen in anorexia nervosa. Nutritional rehabilitation remains the treatment of choice for low bone density in childhood. Calcium supplementation can be considered, although it is likely to have limited value in an underweight child. There is no evidence that high oestrogen doses given as an oral contraceptive improve bone mineral density and they should not be considered without consulting a growth specialist, because of the risks of stunting from premature epiphyseal fusion. Misra et al (2011) have examined physiological oestrogen replacement in adolescent girls, demonstrating increase in bone density of those receiving cyclic transdermal oestrogen for 18 months compared with placebo.

Feeding via nasogastric tube or other method should be considered for children who are unable to tolerate oral refeeding for physical or emotional reasons. It is essential to try to obtain the parents' and/or young person's consent for this - any young person with capacity can consent, although it is uncommon for them to do so in such a situation. In the absence of either, an appropriate legal framework should be invoked. For a young person under the age of 18 and living at home, the options are to use either the Children Act 1989 or the Mental Health Act 1983 (as amended in 2007). Eating disorders are classified as mental disorders under the Mental Health Act and feeding is part of the treatment (i.e. does not require a separate legal mandate). Parental responsibility remains intrinsic to the Children Act, and in some cases it may therefore be preferable to use this legislation rather than the Mental Health Act, which puts professionals effectively in loco parentis.

\section{Target weight}

Patients with anorexia nervosa make every attempt to pin down professionals to giving them a target weight. The clinician's target is for normal growth and development to be restored. Height will 
change during recovery, and thus the target weight range will also change. Lai et al (1994) found that menses resumed at an average of $96 \%$ weight for height, but as for most biological parameters, there is a normal distribution and therefore considerable variation around this. Pelvic ultrasound scan can show whether uterine size and ovarian maturation have progressed (Allan 2010), and is therefore a more sensitive marker of return of hypothalamicpituitary axis function. If pelvic ultrasound is not available, then a target weight range of 95$100 \%$ weight for height is recommended. Weight recovery tends to occur at a rate of about $2-3 \mathrm{~kg}$ per month. In premenarcheal girls and in boys, progress through puberty is a marker that a healthy weight has been regained. Height velocity in growing children should return to normal (or be greater) on restoration to a healthy weight.

\section{Anorexia nervosa and the brain}

The brain in anorexia nervosa is a subject of much interest, given recent advances in neuroscience: two books devoted to eating disorders and the brain have been published in the past few years (Adan 2011; Lask 2011). Neuropsychological and neuroimaging research is being used to better identify those at risk and design treatment interventions. Chui et al (2008) studied brain structure and cognitive function in 66 adolescent girls with relatively long-standing anorexia nervosa compared with healthy controls. They found that participants with anorexia nervosa who remained at low weight had larger lateral ventricles relative to controls, and those who had amenorrhoea or irregular menses showed significant cognitive deficits in a number of domains. Stedal et al (2012) studied the specific neuropsychological profiles of 155 young people with anorexia nervosa in an attempt to better characterise the profile of those at risk and identify patients who might respond to therapies directed at addressing thinking style rather than the primary psychopathology. The participants mainly functioned in the normal range, with particular strengths in verbal fluency and relatively weaker visuospatial memory, setshifting and central coherence.

Central to the research in this area are questions concerning what is primary (i.e. predisposing or risk) and what is secondary to illness and weight loss. Findings have been mixed, depending on the tests performed, the age and duration of illness, and the time to follow-up. Many studies have shown a recovery in performance on weight restoration (for a review, see Jáuregui-Lobera, 2013), although in adults with long-standing anorexia nervosa, Tchanturia et al (2004) showed that performance on set-shifting tasks did not show any improvement following retesting after weight recovery. It may be that a problem with setshifting is a prognostic indicator and is therefore found in treatment-resistant individuals.

Recognition of the role of thinking style and its relationship to anxiety and perfectionism has been the rationale for the development of cognitive remediation therapy (CRT) for anorexia nervosa (Tchanturia 2013a). Trials are ongoing of CRT as an adjunctive treatment and, although it can be difficult to disentangle the effects of weight restoration and improvement in other parameters such as mood (Dahlgren 2013), this is a promising new area for study.

A related area of increasing research is the relationship between 'autistic traits' and anorexia nervosa. Similarities in socioemotional and cognitive domains include difficulties with empathy, set-shifting and global processing. Self-report evidence shows that both adult and adolescent patients (Baron-Cohen 2013; Tchanturia 2013b) have elevated autistic traits relative to controls, particularly in the area of stereotyped and repetitive behaviours (Pooni 2012). Further research is needed in this area to understand the role of autism spectrum traits in the maintenance and treatment response of anorexia nervosa.

\section{Family therapy and parental counselling}

Several studies have reported on family interventions for eating disorders (see 'Therapeutic models', above). Family-based treatment (FBT) is the best studied treatment for the management of anorexia nervosa and therefore has the strongest evidence base for effectiveness in adolescents. It appears to be effective for the majority of adolescents with anorexia nervosa (Lock 2010), with the caveat that those with marked obsessional features or rigidity may need to be treated for longer than the standard 6 months (Lock 2005b). It can be delivered in several formats and doses. Preliminary data suggest that FBT can be disseminated by training and manuals in diverse clinical settings (Couturier 2010). Two systematic reviews, however, reported that FBT was not significantly better or was only marginally better than individual treatment at inducing remission (Gardner 2011; Couturier 2013). Family therapy and parental counselling have been shown to be equally effective (Eisler 2000). A conjoint family approach may be appropriate from the start, particularly for children who are able to articulate their views or conflicts with family members. Parental counselling provides support and advice, using a similar theoretical framework to FBT. In addition to affirming that the parents 
are in charge, parents can explore issues they may be reluctant to discuss in front of their child. Parental self-efficacy is an important predictor of outcome. During FBT, parents can experience an increase in self-efficacy and adolescents a reduction in symptoms. Maternal and paternal self-efficacy scores also predict adolescent outcomes throughout treatment (Robinson 2013).

\section{Group therapy}

Group therapy is an established part of most treatment programmes for adolescents with eating disorders, the focus usually being on the development of self-esteem. Groups for younger children are less well-established. The provision of unstructured time for children to explore peer relationships and to develop freedom of expression can be infinitely more accessible and acceptable to the child than individual therapy, in which a child can feel persecuted and withdraw.

Parents' groups, in both structured (e.g. psychoeducational) and therapeutic/supportive form, can address issues such as coping with rejection, and allow parents to hear from other parents what it can be hard to hear from professionals. Skillsbased workshops (Treasure 2007) for parents and carers of individuals with eating disorders across the age range have also been shown to reduce expressed emotion.

\section{Individual therapy}

The place of individual therapy for older adolescents and those with long-standing illness has long been recognised. In a randomised controlled trial, Lock et al (2010) evaluated the relative efficacy of family-based treatment (FBT) and adolescentfocused individual therapy (AFT) for adolescents with anorexia nervosa in producing full remission. At end of treatment, there were no differences in full remission between FBT and AFT, and both led to considerable improvement. However, those who received AFT were more likely to require hospital admission at some point. Thus, AFT is an alternative when FBT is not feasible or viable.

Fairburn et al (2003) developed a transdiagnostic cognitive-behavioural approach to eating disorder treatment and compared individual and familybased approaches. A 40-week intervention with enhanced cognitive-behavioural therapy (CBT-E) was offered. Participants showed a substantial increase in weight together with a marked decrease in eating disorder psychopathology, which was sustained at 60-week follow-up (Dalle 2013).

For younger children, the role of individual therapy and the therapeutic style adopted depend on a number of factors, not least the availability

of skilled therapists. The nutritional status of the child, as well as their cognitive and emotional development, are important in assessing suitability. Parental support for the therapy is crucial. The focus of work may be to encourage the child to address issues more directly with their parents by rehearsing with the therapist. Individual work should also be considered if family therapy is proving unsuccessful. In such circumstances, previously undisclosed abuse may emerge. Other specific indications for individual work include treatment for concurrent depression, obsessivecompulsive disorder or specific anxieties, such as fear of swallowing or choking. Here, ageappropriate CBT would be the treatment of choice.

It is clear that there is a need therefore to tailor treatments to the individual and family. Le Grange et al (2012) suggest that patients with more severe eating-related psychopathology have better outcomes in a behaviourally targeted family treatment (FBT) than an individually focused approach (AFT).

\section{Bulimia nervosa}

Bulimia nervosa is rare in the premenarcheal age group (Nicholls 2011b). When it does occur (usually around age 13 or 14 and predominantly in girls) the features are fairly similar to those in older adolescents and adults (Box 3). However, purging with laxatives and other medications is less common in the younger age group and secretiveness may be more prominent, since most adolescents continue to be under some sort of parental surveillance. As in adulthood, comorbid depression is often present, and on the rare occasions that in-patient treatment is indicated, depression is more likely to be the reason than the eating behaviour itself. Research among adolescents with bulimia nervosa has lagged behind that among adults, although there is growing evidence that supports

BOX 3 Features of bulimia nervosa

- Persistent preoccupation with eating and recurrent episodes (over a period of months) of binge eating characterised by eating a large amount of food in a short period of time, and a sense of lack of control while eating

- Attempts to counteract the 'fattening' effects of food by compensatory behaviours such as: self-induced vomiting, misuse of purgatives, alternating periods of starvation or excessive exercise, use of drugs such as appetite suppressants, diuretics, thyroid preparations or, in diabetes, misuse of insulin

- Psychopathology consisting of a morbid dread of fatness and setting of a target weight way below what might be considered healthy

- Bulimia nervosa may follow a period of anorexia nervosa, but would only be diagnosed if the patient is no longer significantly underweight

(Adapted from: World Health Organization 1992; American Psychiatric Association 2013) 
the efficacy of family-based interventions and cognitive-behavioural treatments adapted for use with adolescent populations.

Bulimia nervosa is pervaded by a sense of chaos and the first role of the clinician is to establish clear structures and boundaries. The adolescent's behaviour may seem to demand constant supervision, accompaniment to the toilet, supervision after meals and monitoring at school. On the other hand, the adolescent may feel that their parents are being intrusive and become more secretive. Individual therapy can provide structure, containment and privacy for the adolescent, while family work can focus on helping parents negotiate boundaries with their offspring. For example, parents may decide that the adolescent eats three meals a day with the rest of the family, but agree not to accompany him or her to the toilet unless requested to do so. Within this framework, adapted for age where appropriate, adolescents can start to address their own issues in relation to food.

Most patients with bulimia nervosa will be of normal weight or overweight. Despite healthy weights, irregular menses is a common feature and may affect bone density. The most serious medical concern is potassium depletion due to frequent vomiting. Serum potassium is a poor reflection of the body's potassium stores and clinical judgement may be more valuable in deciding whether purging is occurring at a life-threatening level.

Despite advances in cognitive-behavioural techniques for young people and a strong evidence base for adults, there have been only two RCTs comparing their efficacy with family-based treatment in bulimia nervosa (and none in anorexia nervosa). Le Grange et al (2007b) evaluated the relative efficacy of family-based treatment (FBT) and supportive psychotherapy (SPT) for adolescents with bulimia nervosa. Family-based treatment showed a clinical and statistical advantage over SPT at the end of treatment and at 6-month follow-up. Reduction in core bulimic symptoms was also more immediate for patients receiving FBT. However, when Schmidt et al (2007) compared the efficacy and costeffectiveness of family therapy and CBT guided self-care in adolescents with bulimia nervosa or EDNOS, they found that CBT-guided self-care had the advantage of offering a more rapid reduction of bingeing, lower cost and greater acceptability for the adolescents involved, although there were no differences between the treatments overall at 12 months. This suggests that there may be an element of choice for young people about which treatment they would prefer, and some room for clinician judgement based on the level of support from family/parents that the young person may benefit from during their recovery.

\section{Binge eating disorder}

Although binge eating is now classified as an eating disorder, there is as yet no evidence from clinical trials to guide treatment in young people. The initial aim of intervention is typically to address loss of control over eating behaviour with a view to weight stabilisation.

\section{Conclusions}

Although the rates of true eating disorders (anorexia nervosa, bulimia nervosa) in the population are relatively stable, the overall incidence of eating disorders in younger children and adolescents may be increasing and the definitions of eating disorders changing. Eating disorders with onset during childhood and early adolescence, while sharing many common features with lateronset disorders, need to be considered separately from the point of view of recognition, consequences and management. In children, atypical disorders are frequently seen. Epidemiological evidence suggests that primary care clinicians need to be made aware of these disorders, as they are currently under-referred, relative to expected rates, to eating disorder services. Efforts to prevent eating disorders should focus on cases of subthreshold severity.

The principle of care is a comprehensive, multidisciplinary approach, with close collaboration with parents. Evidence-based psychological interventions for new-onset anorexia nervosa and bulimia nervosa are available and effective. A family-based model of treatment is advocated in most cases as the first line, with alternative approaches placing responsibility for change on the young person in the context of development of adolescent autonomy. In-patient treatment is of value both to medically stabilise and to initiate refeeding in acute malnutrition and, less commonly, as a form of intensive therapy. Alternatives to in-patient care are undergoing trials. An evidence-based stepped model of care is currently lacking. The burden on parents/ carers and siblings is high, and recovery typically takes 2-5 years for anorexia nervosa. Bulimia nervosa has higher remission rates, but relapse is more common than for anorexia nervosa. Specific treatments for most atypical eating disorders have not been systematically described or evaluated.

\section{References}

Adan RAH, Kaye WH (eds) (2011) Behavioral Neurobiology of Eating Disorders. Springer. 
Agras WS, Lock J, Brandt H, et al (2014) Comparison of 2 family therapies for adolescent anorexia nervosa: a randomized parallel trial. JAMA Psychiatry, 71: 1279-86.

Allan R, Sharma R, Sangani B, et al (2010) Predicting the weight gain required for recovery from anorexia nervosa with pelvic ultrasonography: an evidence-based approach. European Eating Disorders Review, 18: 43-8.

American Psychiatric Association (2013) Diagnostic and Statistical Manual of Mental Disorders (5th edn) (DSM-5). APA.

Baron-Cohen S, Jaffa T, Davies S, et al (2013) Do girls with anorexia nervosa have elevated autistic traits? Molecular Autism, 4(1): 24.

Bryant-Waugh R, Markham L, Kreipe RE, et al (2010) Feeding and eating disorders in childhood. International Journal of Eating Disorders, 43: 98-111.

Byford S, Barrett B, Roberts C, et al (2007) Economic evaluation of a randomised controlled trial for anorexia nervosa in adolescents. British Journal of Psychiatry, 191: 436-40.

Chui HT, Christensen BK, Zipursky RB, et al (2008) Cognitive function and brain structure in females with a history of adolescent-onset anorexia nervosa. Pediatrics, 122: e426-37.

Court A, Mulder C, Kerr M, et al (2010) Investigating the effectiveness, safety and tolerability of quetiapine in the treatment of anorexia nervosa in young people: a pilot study. Journal of Psychiatric Research, 44: 1027-34.

Couturier J, Isserlin L, Lock J (2010) Family-based treatment for adolescents with anorexia nervosa: a dissemination study. Eating Disorders, 18: 199-209.

Couturier J, Kimber M, Szatmari P (2013) Efficacy of family-based treatment for adolescents with eating disorders: A systematic review and meta-analysis. International Journal of Eating Disorders, 46: 3-11.

Dahlgren CL, Lask B, Landrø NI, et al (2013) Neuropsychological functioning in adolescents with anorexia nervosa before and after cognitive remediation therapy: a feasibility trial. International Journal of Eating Disorders, 46: 576-81.

Dalle GR, Calugi S, Doll HA, et al (2013) Enhanced cognitive behaviour therapy for adolescents with anorexia nervosa: an alternative to family therapy? Behaviour Research and Therapy, 51: R9-12.

Eisler I, Dare C, Russell GF, et al (1997) Family and individual therapy in anorexia nervosa. A 5-year follow-up. Archives of General Psychiatry, 54: $1025-30$.

Eisler I, Dare C, Hodes M, et al (2000) Family therapy for adolescent anorexia nervosa: the results of a controlled comparison of two family interventions. Journal of Child Psychology and Psychiatry, 41: 727-36.

Fairburn CG, Cooper Z, Shafran R (2003) Cognitive behaviour therapy for eating disorders: a 'transdiagnostic' theory and treatment. Behaviour Research and Therapy, 41: 509-28.

Field AE, Sonneville KR, Micali N, et al (2012) Prospective association of common eating disorders and adverse outcomes. Pediatrics, 130: e289-95.

Fitzpatrick K, Moye A, Hoste RR, et al (2010) Adolescent focused psychotherapy for adolescents with anorexia nervosa. Journal of Contemporary Psychotherapy, 40: 31-9.

Flament MF, Bissada H, Spettigue W (2012) Evidence-based pharmacotherapy of eating disorders. International Journal of Neuropsychopharmacology, 15: 189-207.

Gardner J, Wilkinson P (2011) Is family therapy the most effective treatment for anorexia nervosa? Psychiatria Danubina, 23 (suppl. 1): S175-7.

Gowers S, Green L (2009) Eating Disorders: Cognitive Behavioural Therapy with Children and Young People. Taylor \& Francis.

Gowers SG, Clark A, Roberts C, et al (2007) Clinical effectiveness of treatments for anorexia nervosa in adolescents: randomised controlled trial. British Journal of Psychiatry, 191: 427-35.

Hagman J, Gralla J, Sigel E, et al (2011) A double-blind, placebo-controlled study of risperidone for the treatment of adolescents and young adults with anorexia nervosa: a pilot study. Journal of the American Academy of Child \& Adolescent Psychiatry, 50: 915-24.
Herpertz-Dahlmann B, Muller B, Herpertz S, et al (2001) Prospective 10-year follow-up in adolescent anorexia nervosa - course, outcome, psychiatric comorbidity, and psychosocial adaptation. Journal of Child Psychology and Psychiatry, 42: 603-12.

Herpertz-Dahlmann B, Schwarte R, Krei M, et al (2014) Day-patient treatment after short inpatient care versus continued inpatient treatment in adolescents with anorexia nervosa (ANDI): a multicentre, randomised, open-label, non-inferiority trial. Lancet, 383: 1222-9.

House J, Schmidt U, Craig M, et al (2012) Comparison of specialist and nonspecialist care pathways for adolescents with anorexia nervosa and related eating disorders. International Journal of Eating Disorders, 45: 949-56.

Hudson LD, Nicholls DE, Lynn RM, et al (2012) Medical instability and growth of children and adolescents with early onset eating disorders. Archives of Disease in Childhood, 97: 779-84.

Jáuregui-Lobera I (2013) Neuropsychology of eating disorders: 19952012. Neuropsychiatric Disease and Treatment, 9: 415-30.

Kafantaris V, Leigh E, Hertz S, et al (2011) A placebo-controlled pilot study of adjunctive olanzapine for adolescents with anorexia nervosa. Journal of Child and Adolescent Psychopharmacology, 21: 207-12.

Keel PK, Brown TA (2010) Update on course and outcome in eating disorders. International Journal of Eating Disorders, 43: 195-204.

Kotler LA, Cohen P, Davies M, et al (2001) Longitudinal relationships between childhood, adolescent, and adult eating disorders. Journal of the American Academy of Child \& Adolescent Psychiatry, 40: 1434-40.

Lai KY, de Bruyn R, Lask B, et al (1994) Use of pelvic ultrasound to monitor ovarian and uterine maturity in childhood onset anorexia nervosa. Archives of Disease in Childhood, 71: 228-31.

Lask B, Frampton I (eds) (2011) Eating Disorders and the Brain. WileyBlackwell.

Le Grange D, Lock J (2007a) Treating Bulimia in Adolescents: A FamilyBased Approach. Guilford Press.

Le Grange D, Crosby RD, Rathouz PJ, et al (2007b) A randomized controlled comparison of family-based treatment and supportive psychotherapy for adolescent bulimia nervosa. Archives of General Psychiatry, 64: 1049-56. Le Grange D, Crosby RD, Lock J (2008) Predictors and moderators of outcome in family-based treatment for adolescent bulimia nervosa. Journal of the American Academy of Child \& Adolescent Psychiatry, 47 : 464-70.

Le Grange D, Lock J, Loeb K, et al (2010) Academy for Eating Disorders position paper: the role of the family in eating disorders. International Journal of Eating Disorders, 43: 1-5.

Le Grange D, Lock J, Agras WS, et al (2012) Moderators and mediators of remission in family-based treatment and adolescent focused therapy for anorexia nervosa. Behaviour Research and Therapy, 50: 85-92.

Lock J, Le Grange D, Agras S, et al (2000) Treatment Manual for Anorexia Nervosa. Guilford Press.

Lock J, Walker LR, Rickert VI, et al (2005a) Suicidality in adolescents being treated with antidepressant medications and the black box label: position paper of the Society for Adolescent Medicine. Journal of Adolescent Health, 36: 92-3.

Lock J, Agras WS, Bryson S, et al (2005b) A comparison of short- and long-term family therapy for adolescent anorexia nervosa. Journal of the American Academy of Child \& Adolescent Psychiatry, 44: 632-9.

Lock J, Le Grange D, Agras WS, et al (2010) Randomized clinical trial comparing family-based treatment with adolescent-focused individual therapy for adolescents with anorexia nervosa. Archives of General Psychiatry, 67: 1025-32.

Lock J (2015) An update on evidence-based psychosocial treatments for eating disorders in children and adolescents. Journal of Clinical Child and Adolescent Psychology, Jan 12: PMID 25580937 [Epub ahead of print].

Lucas AR, Beard CM, O'Fallon WM, et al (1991) 50-year trends in the incidence of anorexia nervosa in Rochester, Minn.: a population-based study. American Journal of Psychiatry, 148: 917-22.

Madden S, Miskovic-Wheatley J, Wallis A, et al (2014) A randomized controlled trial of in-patient treatment for anorexia nervosa in medically

\section{MCO answers \\ 1 e $\quad 2 d \quad 3$ e $\quad 4$ b $\quad 5 c$}


unstable adolescents. Psychological Medicine, 14: Jul 14: PMID: 25017941 [Epub ahead of print].

Marcus MD, Wildes JE (2009) Obesity: is it a mental disorder? International Journal of Eating Disorders, 42: 739-53.

Misra M, Katzman D, Miller KK, et al (2011) Physiologic estrogen replacement increases bone density in adolescent girls with anorexia nervosa. Journal of Bone and Mineral Research, 26: 2430-8.

National Collaborating Centre for Mental Health (2004) Eating Disorders. Core Interventions in the Treatment and Management of Anorexia Nervosa, Bulimia Nervosa and Related Eating Disorders (Clinical Guideline 9). National Institute for Clinical Excellence.

Nesbitt S (2005) Hunger for Understanding: A Workbook for Helping Young People to Understand and Overcome Anorexia Nervosa. WileyBlackwell.

Nicholls D, Hudson L, Mahomed F (2011a) Managing anorexia nervosa. Archives of Disease in Childhood, 96: 977-82.

Nicholls DE, Lynn R, Viner RM (2011b) Childhood eating disorders: British national surveillance study. British Journal of Psychiatry, 198: 295-301.

Peebles R, Hardy KK, Wilson JL, et al (2010) Are diagnostic criteria for eating disorders markers of medical severity? Pediatrics, 125 : e1193-201.

Pereira T, Lock J, Oggins J (2006) Role of therapeutic alliance in family therapy for adolescent anorexia nervosa. International Journal of Eating Disorders, 39: 677-84.

Pooni J, Ninteman A, Bryant-Waugh R, et al (2012) Investigating autism spectrum disorder and autistic traits in early onset eating disorder International Journal of Eating Disorders, 45: 583-91.

Robinson AL, Strahan E, Girz L, et al (2013) 'I know I can help you': parental self-efficacy predicts adolescent outcomes in family-based therapy for eating disorders. European Eating Disorders Review, 21: 108-14.

Royal College of Psychiatrists (2012) Junior MARSIPAN: Management of Really Sick Patients under 18 with Anorexia Nervosa (College Report CR168). Royal College of Psychiatrists.

Schmidt U, Lee S, Beecham J, et al (2007) A randomized controlled trial of family therapy and cognitive behavior therapy guided self-care for adolescents with bulimia nervosa and related disorders. American Journal of Psychiatry, 164: 591-8.
Shepperd S, Doll H, Gowers S, et al (2009) Alternatives to inpatient mental health care for children and young people. Cochrane Database of Systematic Reviews, 2: CD006410.

Stedal K, Rose M, Frampton I, et al (2012) The neuropsychological profile of children, adolescents, and young adults with anorexia nervosa. Archives of Clinical Neuropsychology, 27: 329-37.

Stice E, Marti CN, Rohde P (2013) Prevalence, incidence, impairment, and course of the proposed DSM-5 eating disorder diagnoses in an 8-year prospective community study of young women. Journal of Abnormal Psychology, 122: 445-57.

Tan JO, Hope T, Stewart A, et al (2003) Control and compulsory treatment in anorexia nervosa: the views of patients and parents. International Journal of Law and Psychiatry, 26: 627-45.

Tchanturia K, Morris RG, Anderluh MB, et al (2004) Set shifting in anorexia nervosa: an examination before and after weight gain, in full recovery and relationship to childhood and adult OCPD traits. Journal of Psychiatric Research, 38: 545-52.

Tchanturia K, Lloyd S, Lang K (2013a) Cognitive remediation therapy for anorexia nervosa: current evidence and future research directions. International Journal of Eating Disorders, 46: 492-5.

Tchanturia K, Smith E, Weineck F, et al (2013b) Exploring autistic traits in anorexia: a clinical study. Molecular Autism, 4: 44.

Treasure J, Smith G, Crane A (2007) Skills-Based Learning for Caring for a Loved One with an Eating Disorder: The New Maudsley Method. Routledge.

Troupp C (2013) Individual psychotherapy. In Eating Disorders in Childhood and Adolescence (4th edn) (eds B Lask, R Bryant-Waugh): 281-300. Routledge.

Uher R, Rutter M (2012) Classification of feeding and eating disorders: review of evidence and proposals for ICD-11. World Psychiatry, 11: 80-92.

Watkins B (2013) Cognitive behavioural approaches. In Eating Disorders in Childhood and Adolescence (4th edn) (eds B Lask, R Bryant-Waugh): 258-80. Routledge.

Watkins B, Cooper PJ, Lask B (2012) History of eating disorder in mothers of children with early onset eating disorder or disturbance. European Eating Disorders Review, 20: 121-5.

World Health Organization (1992) The ICD-10 Classification of Mental and Behavioural Disorders: Clinical Descriptions and Diagnostic Guidelines. WHO

\section{MCOs}

Select the single best option for each question stem

\section{As regards the classification and epidemiology of eating disorders:}

a $50 \%$ of adolescents respond to treatment within 6 months

b in-patient treatment is associated with a better prognosis

c axis 1 comorbidity does not affect outcome

d recovered patients display significant deficiencies in psychosocial functioning

e obesity is not classified as an eating disorder.

\section{As regards therapeutic models for} childhood eating disorders:

a behavioural techniques have no role in treatment

b patients with marked obsessional features are not suitable for family-based treatments

c parents should not be afforded any responsibilities in the treatment process

$\mathrm{d}$ the therapeutic alliance is not associated with treatment outcome e parental self-efficacy shows no association with treatment outcome.

3 As regards pharmacological treatments of anorexia nervosa:

a quetiapine use has been shown to improve weight restoration

b risperidone use has been shown to improve weight restoration

c olanzapine use has been shown to improve weight restoration

d risperidone use has been shown to improve psychological function

e quetiapine use has been shown to improve psychological function.

4 Family-based treatments for bulimia nervosa:

a show a more rapid reduction in core bulimic symptoms than CBT

b show a more rapid reduction in core bulimic symptoms than SPT

c are more effective overall than CBT at 12-month follow-up $\mathrm{d}$ are more cost-effective than CBT and SPT

e are more acceptable to adolescent patients.

5 Research into the delivery of eating disorder services suggests that:

a in-patient care is more efficacious than outpatient services

b specialist services are more efficacious

c specialist services are more cost-effective

d out-patient treatments are more cost-effective

e there is no role for general CAMHS services in the management of eating disorders. 